\title{
Eficiência de fosfato natural reativo aplicado em misturas com superfosfato triplo em milho e soja
}

\author{
Vinícius Ide Franzini(1), Takashi Muraoka ${ }^{(2)}$, Héctor Manuel Coraspe-León ${ }^{(3)}$ e Fernanda Latanze Mendes ${ }^{(1)}$ \\ (1)Universidade de São Paulo, Escola Superior de Agricultura Luiz de Queiroz, Departamento de Ciência do Solo, Caixa Postal 9, CEP 13418-900 Piracicaba, SP. \\ E-mail: vifranzi@esalq.usp.br, flmendes@cena.usp.br ${ }^{(2)}$ Universidade de São Paulo, Centro de Energia Nuclear na Agricultura, Caixa Postal 96, \\ CEP 13416-000 Piracicaba, SP. E-mail: muraoka@cena.usp.br ${ }^{(3)}$ Instituto Nacional de Investigaciones Agrícolas, Pampanito, Trujillo, Venezuela. \\ E-mail: hcoraspe@inia.gov.ve
}

Resumo - O objetivo deste trabalho foi avaliar o efeito do modo de aplicação do superfosfato triplo (SFT), do fosfato natural reativo de Gafsa e da mistura entre essas fontes no aproveitamento do fósforo (P) por plantas de milho e de soja. Os experimentos foram realizados em casa de vegetação, e as plantas foram cultivadas em vasos com Latossolo Vermelho-Amarelo distrófico. As fontes fosfatadas foram aplicadas isoladamente ou em misturas (proporção 1:1), à dose de $90 \mathrm{mg} \mathrm{kg}^{-1}$ de $\mathrm{P}$, de modo não localizado ou localizado no solo. Para a quantificação do $\mathrm{P}$ absorvido pelas plantas das diferentes fontes, empregaram-se tratamentos com o solo e com o SFT marcados com ${ }^{32} \mathrm{P}$. O aproveitamento do fósforo do superfosfato triplo aplicado de forma localizada $(9,8 \%)$ foi superior ao da não localizada (7\%). A aplicação não localizada do fosfato natural reativo proporcionou maior aproveitamento do fósforo $(3,8 \%)$, em comparação à localizada $(0,5 \%)$. Em comparação à aplicação isolada do fosfato de Gafsa, o aproveitamento do fósforo pelas plantas de milho e soja aumentou quando essa fonte foi aplicada como mistura compactada; em presença do superfosfato triplo, a aplicação do fosfato natural reativo de Gafsa de modo não localizado proporcionou maior aproveitamento do P pelas plantas de soja. O aproveitamento do fósforo do fosfato natural de Gafsa aumenta quando aplicado ao solo com o superfosfato triplo, e esse efeito depende do método de aplicação das fontes fosfatadas.

Termos para indexação: Glycine max, Zea mays, diluição isotópica, fertilizante fosfatado, fosfato natural de Gafsa, Latossolo.

\section{Efficiency of reactive phosphate rock applied in mixtures with triple superphosphate in maize and soybean}

\begin{abstract}
The objective of this work was to evaluate the effects of methods of applying triple superphosphate (TSP), Gafsa reactive phosphate rock and mixtures between these sources on phosphorus $(\mathrm{P})$ utilization by maize and soybean plants. The experiments were carried out in a greenhouse, and the plants were grown in pots containing Typic Haplustox soil. The phosphorus sources were applied isolatedly or mixed (1:1 ratio) in a P rate of $90 \mathrm{mg} \mathrm{kg}^{-1}$, in a non-localized or in a localized manner in the soil. To quantify plant-absorbed phosphorus from the different sources, treatments with soil and TSP labeled with ${ }^{32} \mathrm{P}$ were used. Phosphorus utilization from triple superphosphate applied locally was superior (9.8\%) to the P utilization of this source applied non locally to the soil (7\%). Inversely, better results with Gafsa phosphate rock occurred with P applied non locally to soil (3.8\%), compared to the values obtained when the fertilizer was applied locally $(0.5 \%)$. The phosphorus utilization from the Gafsa phosphate rock by maize and soybean plants increased when it was applied as compacted triple superphosphate and Gafsa phosphate rock mixture. In the presence of triple superphosphate, the soil-incorporated application of Gafsa phosphate rock led to greater utilization of phosphorus by soybean plants. The utilization of Gafsa phosphate rock phosphorus increases when it is applied together with triple superphosphate; and this effect depends on the methods of application of these phosphate sources.
\end{abstract}

Index terms: Glycine max, Zea mays, isotopic dilution, phosphorus fertilizers, Gafsa phosphate rock, Oxisol, radioisotopic labelling.

\section{Introdução}

No Brasil, as culturas de soja e milho ocupam, respectivamente, o primeiro e segundo lugar em área e em valor de produção entre as culturas graníferas. Na safra 2007/2008, as áreas cultivadas com soja e milho foram de 21,33 e 14,71 milhões de hectares com produção de 60,05 e 58,59 milhões de toneladas, respectivamente (Companhia Nacional de Abastecimento, 2008).

$\mathrm{O}$ fósforo $(\mathrm{P})$ é o nutriente mais limitante para a produtividade de biomassa em solos tropicais, e a baixa reversibilidade da adsorção desse elemento ao solo (Novais \& Smyth, 1999) diminui a eficiência no seu aproveitamento pelas plantas em solos altamente intemperizados. 
Os fosfatos de origem sedimentar, como o de Gafsa (proveniente da Tunísia), possuem alta reatividade (Syers et al., 1986). No Brasil, esse fosfato é comercializado como fonte alternativa, de custo mais baixo, e tem apresentado maior efeito residual no solo, quando comparado aos superfosfatos (Sousa et al., 2008).

Entre as estratégias definidas por Goedert \& Sousa (1984), para aumentar a eficiência das adubações fosfatadas, deve-se atentar para o método mais adequado de aplicação das fontes de fósforo. A aplicação de fontes de fósforo com elevada solubilidade juntamente com fontes alternativas, com menor solubilidade e custo, pode ser uma maneira de melhorar a eficiência das fontes menos solúveis, como os fosfatos reativos. Esse aumento do aproveitamento do $\mathrm{P}$ dos fosfatos naturais com a aplicação conjunta de fontes solúveis pode ser decorrente da promoção de maior crescimento do sistema radicular no estágio inicial de desenvolvimento das culturas, na presença de uma forma solúvel de $\mathrm{P}$ no solo (Chien et al., 1996). A solubilização parcial do fosfato natural reativo pela acidez gerada da hidrólise dos superfosfatos no solo (Mokwunye \& Chien, 1980) pode ser outra explicação para o aumento da eficiência das fontes alternativas de fósforo com a aplicação conjunta. Para que isso ocorra, no entanto, as duas fontes devem ser aplicadas em contato (no mesmo grânulo). Atualmente, no Brasil, existem fontes de P em que há mistura de fosfato natural reativo com fontes fosfatadas totalmente aciduladas.

O objetivo deste trabalho foi avaliar o efeito do modo de aplicação do superfosfato triplo, do fosfato natural reativo de Gafsa e da mistura entre essas fontes no aproveitamento do fósforo $(\mathrm{P})$ por plantas de milho e de soja.

\section{Material e Métodos}

Dois experimentos foram desenvolvidos em casa de vegetação do Centro de Energia Nuclear na Agricultura, a $22^{\circ} 42^{\prime} 30^{\prime \prime} \mathrm{S}, 47^{\circ} 38^{\prime} 01^{\prime \prime} \mathrm{W}$ e a $554 \mathrm{~m}$ de altitude, em Piracicaba, SP. Plantas de milho e soja foram cultivadas em vasos com amostras de terra da camada superficial (0 a 0,2 m) de um Latossolo Vermelho-Amarelo distrófico (Santos et al., 2006), coletadas no Município de Piracicaba, SP.

O solo apresentou teores de argila, silte e areia de 280, 70 e $650 \mathrm{~g} \mathrm{~kg}^{-1}$, respectivamente (Camargo et al., 1986), e as seguintes características químicas: pH $\left(0,01\right.$ mol L $\left.\mathrm{L}^{-1} \mathrm{CaCl}_{2}\right), 4,7$; matéria orgânica, $20 \mathrm{~g} \mathrm{dm}^{-3}$; P extraído por resina, $6 \mathrm{mg} \mathrm{dm}^{-3}$; $\mathrm{K}, \quad 0,8 \quad \mathrm{mmol}_{\mathrm{c}} \mathrm{dm}^{-3} ; \mathrm{Ca}, \quad 12,9 \mathrm{mmol}_{\mathrm{c}} \mathrm{dm}^{-3}$; $\mathrm{Mg}, 6,4 \mathrm{mmol}_{\mathrm{c}} \mathrm{dm}^{-3} ; \mathrm{H}+\mathrm{Al}, 31,2 \mathrm{mmol}_{\mathrm{c}} \mathrm{dm}^{-3} ; \mathrm{CTC}$, 51,3 $\mathrm{mmol}_{\mathrm{c}} \mathrm{dm}^{-3}$; soma de bases, 20,1 $\mathrm{mmol}_{\mathrm{c}} \mathrm{dm}^{-3}$; saturação por bases, 39,2\% (Raij et al., 2001).

Após a aplicação de calcário (PRNT =90,4\%), para elevar a saturação por bases a $70 \%$ para o cultivo de milho e $60 \%$ para o de soja (Raij et al., 1996), o solo foi incubado durante 30 dias, e a umidade foi mantida em aproximadamente $70 \%$ da capacidade de campo.

O delineamento experimental usado em ambos os experimentos foi o inteiramente casualizado, com quatro repetições. As fontes de $\mathrm{P}$ utilizadas foram o superfosfato triplo (SFT) e o fosfato natural reativo de Gafsa (FN), na dose de $90 \mathrm{mg} \mathrm{kg}^{-1}$ de P.

Em ambos os experimentos, para a quantificação do $\mathrm{P}$ absorvido pelas plantas, proveniente do $\mathrm{FN}$, empregaram-se tratamentos com o solo marcado com ${ }^{32} \mathrm{P}$ (método indireto de marcação) e tratamentos adicionais com o SFT também marcado com ${ }^{32} \mathrm{P}$ (método direto de marcação). Nos tratamentos em que o solo foi marcado com ${ }^{32} \mathrm{P}$, aplicou-se o SFT não marcado e vice-versa. A vantagem dessa técnica com o radioisótopo ${ }^{32} \mathrm{P}$ é a possibilidade de distinguir, do total de $\mathrm{P}$ acumulado na planta, as frações de $\mathrm{P}$ que foram absorvidos do FN ou do SFT. Para isso, as quantidades de $\mathrm{P}$ na planta provenientes do SFT e do FN e o aproveitamento do $\mathrm{P}$ dessas fontes foram calculados pelas equações do método da diluição isotópica de ${ }^{32} \mathrm{P}$, conforme descrito por Chien et al. (1996) e Franzini et al. (2009). O fósforo acumulado (P) foi determinado pela equação $\mathrm{P}=[\mathrm{P}] \times \mathrm{MSPA}$, em que $\mathrm{P}$ é o fósforo acumulado (mg), $[\mathrm{P}]$ é a concentração de fósforo $\left(\mathrm{g} \mathrm{kg}^{-1}\right)$, e MSPA é a massa de matéria seca da parte aérea $(\mathrm{g})$.

A atividade específica do ${ }^{32} \mathrm{P}$ nas plantas foi determinada pela equação $\mathrm{AE}={ }^{32} \mathrm{P} /{ }^{31} \mathrm{P}$, em que $\mathrm{AE}$ é a atividade específica (DPM $\left.\mu \mathrm{gP}^{-1}\right) ;{ }^{32} \mathrm{P}$ é a atividade do radioisótopo na planta (DPM); ${ }^{31} \mathrm{P}$ é o conteúdo de $\mathrm{P}$ na planta ( $\mu$ g de P planta ${ }^{-1}$ ).

A fração do $\mathrm{P}$ absorvido do $\mathrm{FN}\left(\mathrm{F}_{\mathrm{FN}}\right)$ e o P absorvido do $\mathrm{FN}\left(\mathrm{P}_{\mathrm{FN}}\right)$ foram determinados pelas equações: $\mathrm{F}_{\mathrm{FN}}=1-\left[\mathrm{AE}_{\mathrm{P}(\mathrm{FN}+\text { solo })} / \mathrm{AE}_{\mathrm{P}(\text { solo })}\right] ; \mathrm{P}_{\mathrm{FN}}=\mathrm{P}_{(\mathrm{FN}+\text { solo })} \times \mathrm{F}_{\mathrm{FN}}, \mathrm{em}$ que: $\mathrm{AE}_{\mathrm{P}(\mathrm{FN}+\text { solo) }}$ é a atividade específica do $\mathrm{P}$ absorvido do $\mathrm{FN}+$ solo, na planta; $\mathrm{AE}_{\mathrm{p}(\text { solo) }}$ é a atividade específica 
do $\mathrm{P}$ absorvido do solo (testemunha), na planta; $\mathrm{P}_{(\mathrm{FN}+\text { solo })}$ é o P absorvido do FN + solo.

A fração do $\mathrm{P}$ absorvido do SFT ( $\left.\mathrm{F}_{\mathrm{SFT}}\right)$, o P absorvido do SFT ( $\left.\mathrm{P}_{\mathrm{SFT}}\right)$ e o P absorvido do solo, na presença do SFT $\left(\mathrm{P}_{\text {solo (SFT) }}\right)$, foram determinados pelas equações: $\mathrm{F}_{\mathrm{SFT}}=\left[\mathrm{AE}_{\mathrm{P}(\mathrm{SFT}+\text { solo })} / \mathrm{AE}_{\mathrm{P}(\mathrm{SFT})}\right] ; \mathrm{P}_{\mathrm{SFT}}=\mathrm{P}_{(\mathrm{SFT}+\text { solo })} \times \mathrm{F}_{\mathrm{SFT}} ;$ $\mathrm{P}_{\text {solo (SFT) }}=\mathrm{P}_{(\mathrm{SFT}+\text { solo })}-\mathrm{P}_{\mathrm{SFT}}$, em que: $\mathrm{AE}_{\mathrm{P}(\mathrm{SFT}+\text { solo })}$ é a atividade específica do $\mathrm{P}$ absorvido do SFT + solo; $\mathrm{AE}_{\mathrm{P}(\mathrm{SFT})}$ é a atividade específica do $\mathrm{P}$ absorvido do SFT; $\mathrm{P}_{\text {(SFT + solo) }}$ é o P absorvido do SFT + solo; $\mathrm{P}_{\mathrm{SFT}}$ é o $\mathrm{P}$ absorvido do SFT.

A fração de $\mathrm{P}$ absorvido do SFT em presença de FN $\left(\mathrm{F}_{\mathrm{SFT}(\mathrm{FN})}\right)$, o $\mathrm{P}$ absorvido do SFT em presença de $\mathrm{FN}\left(\mathrm{P}_{\mathrm{SFT}(\mathrm{FN})}\right)$, o $\mathrm{P}$ absorvido do $\mathrm{FN}+$ solo em presença de SFT $\left(\mathrm{P}_{(\mathrm{FN}+\text { solo)(SFT) }}\right)$, e o P absorvido do FN em presença de SFT $\left(\mathrm{P}_{\mathrm{FN}(\mathrm{SFT})}\right)$ foram determinados pelas equações: $\mathrm{F}_{\mathrm{SFT}(\mathrm{FN})}=\mathrm{AE}_{\mathrm{P}(\mathrm{FN}+\mathrm{SFT}+\text { solo })} / \mathrm{AE}_{\mathrm{P}(\mathrm{SFT})} ; \mathrm{P}_{\mathrm{SFT}(\mathrm{FN})}$ $=\mathrm{P}_{(\mathrm{FN}+\mathrm{SFT}+\text { solo })} \mathrm{F}_{\mathrm{SFT}(\mathrm{FN})} ; \mathrm{P}_{(\mathrm{FN}+\text { solo) }(\mathrm{SFT})}=\mathrm{P}_{(\mathrm{FN}+\mathrm{SFT}+\text { solo })}-$ $\mathrm{P}_{\mathrm{SFT}(\mathrm{FN})} ; \mathrm{P}_{\mathrm{FN}(\mathrm{SFT})}=\mathrm{P}_{(\mathrm{FN}+\text { solo)(SFT) }}-\mathrm{P}_{\text {solo (FN }+\mathrm{SFT})}$, em que: $\mathrm{AE}_{\mathrm{P}(\mathrm{FN}+\mathrm{SFT}+\text { solo })}$ é a atividade específica do $\mathrm{P}$ absorvido do $\mathrm{FN}+\mathrm{SFT}+$ solo; $\mathrm{AE}_{\mathrm{P}(\mathrm{SFT})}$ é a atividade específica do P absorvido do fertilizante SFT; $\mathrm{P}_{(\mathrm{FN}+\mathrm{SFT}+\text { solo) }}$ é o $\mathrm{P}$ absorvido do FN + SFT + solo; $\left.\mathrm{P}_{\text {solo( }(\mathrm{FN}}+\mathrm{SFT}\right)$ é o $\mathrm{P}$ absorvido do solo em presença do (FN + SFT).

O aproveitamento $[\mathrm{Ap}(\%)]$ do $\mathrm{P}$ do fertilizante ( $\mathrm{FN} \mathrm{ou}$ SFT), ou eficiência da fonte fosfatada para as plantas foi determinado pela equação: $\mathrm{Ap}(\%)=100\left(\mathrm{P}_{\mathrm{PPF}} / \mathrm{P}_{\text {aplicado }}\right)$, em que: $\mathrm{P}_{\mathrm{PPF}}$ é o $\mathrm{P}$ na planta proveniente do fertilizante (FN ou SFT) (mg); $\mathrm{P}_{\text {aplicado }}$ é a quantidade de P aplicada ao solo proveniente do fertilizante (FN ou SFT) (mg).

A marcação do solo com ${ }^{32} \mathrm{P}$, nos dois experimentos, foi realizada com $20 \mathrm{~g}$ de areia, que inicialmente receberam 7,4 MBq de ${ }^{32} \mathrm{P}$ livre de carregador. A areia foi aplicada uniformemente a $1.980 \mathrm{~g}$ de solo mediante agitação vigorosa de saco plástico contendo o solo e a areia marcada.

No primeiro experimento, plantas de milho foram cultivadas em vasos com $2 \mathrm{~kg}$ de terra, em dez tratamentos com o solo marcado com ${ }^{32} \mathrm{P}$, cinco deles constituídos pela testemunha (sem aplicação de P) e aplicação isolada das fontes fosfatadas - SFT localizado, SFT não localizado, FN localizado, FN não localizado -; e mais cinco tratamentos com as misturas entre as duas fontes de P - SFT localizado + FN localizado (em contato no mesmo sulco), SFT localizado + FN não localizado, SFT não localizado + FN localizado, SFT não localizado + FN não localizado e SFT + FN no mesmo grânulo (mistura compactada, que foi aplicada de modo localizado ao solo). Neste experimento, foram utilizados sete tratamentos adicionais com o SFT marcado com ${ }^{32} \mathrm{P}$ ( $\left.{ }^{32} \mathrm{P}-\mathrm{SFT}\right)$ e solo não marcado: ${ }^{32} \mathrm{P}-\mathrm{SFT}$ localizado, ${ }^{32} \mathrm{P}-\mathrm{SFT}$ não localizado, ${ }^{32} \mathrm{P}-\mathrm{SFT}$ localizado $+\mathrm{FN}$ localizado (em contato no mesmo sulco), ${ }^{32} \mathrm{P}$-SFT localizado + FN não localizado, ${ }^{32} \mathrm{P}-\mathrm{SFT}$ não localizado + FN localizado, ${ }^{32} \mathrm{P}-\mathrm{SFT}$ não localizado $+\mathrm{FN}$ não localizado e ${ }^{32} \mathrm{P}-\mathrm{SFT}$ + FN no mesmo grânulo (mistura compactada, que foi aplicada de modo localizado ao solo). O SFT e a mistura compactada foram aplicadas em grânulo e o FN na formulação farelada.

As fontes de $\mathrm{P}$ que apresentaram valores superiores de aproveitamento do P do SFT e do FN, pelo milho, foram selecionadas para um segundo experimento, em que se utilizaram plantas de soja como cultura teste.

As plantas de soja foram cultivadas em vasos com $2,5 \mathrm{~kg}$ de terra, e o experimento foi constituído de seis tratamentos com o solo marcado com ${ }^{32} \mathrm{P}$ : testemunha (sem aplicação de P), SFT localizado, FN não localizado, e as misturas entre as duas fontes de $\mathrm{P}$, que foram SFT localizado + FN localizado (em contato no mesmo sulco), SFT localizado + FN não localizado e SFT + FN no mesmo grânulo (mistura compactada, que foi aplicada de modo localizado ao solo). Neste experimento, foram utilizados quatro tratamentos adicionais com o SFT marcado com ${ }^{32} \mathrm{P}\left({ }^{32} \mathrm{P}-\mathrm{SFT}\right)$ e solo não marcado: ${ }^{32} \mathrm{P}-\mathrm{SFT}$ localizado, ${ }^{32} \mathrm{P}-\mathrm{SFT}$ localizado + FN localizado (em contato no mesmo sulco), ${ }^{32} \mathrm{P}-\mathrm{SFT}$ localizado + FN não localizado e ${ }^{32} \mathrm{P}-\mathrm{SFT}+\mathrm{FN}$ no mesmo grânulo (mistura compactada, que foi aplicada de modo localizado ao solo).

A proporção utilizada nas misturas das duas fontes de $\mathrm{P}$ foi de 1:1, tendo-se considerado o teor total de $\mathrm{P}$ dessas fontes, ou seja, $45 \mathrm{mg} \mathrm{kg}^{-1}$ de P como SFT e $45 \mathrm{mg} \mathrm{kg}^{-1}$ de P como FN.

$\mathrm{Na}$ aplicação realizada de forma não localizada para simular a aplicação a lanço e posterior incorporação, os sacos de plástico com o solo e os fertilizantes foram agitados vigorosamente visando à homogenização. Já a aplicação localizada foi realizada com a distribuição das fontes de $\mathrm{P}$ em sulcos, a aproximadamente $5 \mathrm{~cm}$ de profundidade.

O termo mistura compactada, neste trabalho, referese à fonte de $\mathrm{P}$ obtida pela mistura do SFT com o FN e água deionizada, ou seja, a mistura dessas duas fontes fosfatadas no mesmo grânulo. Esse processo foi denominado de compactação úmida e foi realizado 
com o objetivo de se comparar o aproveitamento do $\mathrm{P}$ da mistura compactada, com o do tratamento em que o SFT e o FN foram aplicados em contato físico, no mesmo sulco, mas não no mesmo grânulo. Assim, foi possível verificar se ocorre interação química entre essas duas fontes de $\mathrm{P}$ e o aumento do aproveitamento do P do FN (efeito químico).

Para o preparo dessa mistura, o SFT em grânulos foi moído e peneirado (peneira de $0,5 \mathrm{~mm}$ ). O FN de Gafsa também foi peneirado (peneira de $0,5 \mathrm{~mm}$ ), e foram pesadas as quantidades correspondentes de SFT e FN misturadas e umedecidas com água deionizada, para se obter uma mistura homogênea. Essas misturas foram secas em estufa com circulação de ar a $90^{\circ} \mathrm{C}$ por 24 horas e, após esse período, as misturas foram fragmentadas em grânulos com diâmetro entre 2 e $4 \mathrm{~mm}$, semelhantes aos do fertilizante SFT.

Em ambos os experimentos, da mesma forma que no processo realizado no preparo da mistura compactada, o SFT também foi moído e peneirado (peneira de 0,5 mm), para a aplicação nos outros tratamentos. Em seguida, o SFT foi umedecido com água deionizada, seco em estufa a $90^{\circ} \mathrm{C}$ por 24 horas e quebrado em grânulos de tamanho similar ao do fertilizante SFT (diâmetro entre 2 e $4 \mathrm{~mm}$ ). Esse processo foi realizado tanto para o fertilizante SFT não marcado como para ${ }^{32} \mathrm{P}-\mathrm{SFT}$ marcado, que recebeu a solução radioativa (atividade de $55 \mathrm{KBq}$ ${ }^{32} \mathrm{P} \mathrm{mg}^{-1}$ de P).

Três sementes de milho (híbrido Pioneer 30F33) foram semeadas por vaso com $2 \mathrm{~kg}$ de solo e desbastadas, tendo-se deixado uma planta, seis dias após a emergência. Cinco sementes de soja (cultivar IAC 18) foram semeadas por vaso (2,5 $\mathrm{kg}$ de solo) e desbastadas, tendo-se deixado duas plantas, cinco dias após a emergência.

Nos dois experimentos, nitrogênio $(\mathrm{N})$ e potássio (K) foram aplicados nas doses $150 \mathrm{mg} \mathrm{kg}^{-1} \mathrm{de} \mathrm{N}$, como sulfato de amônio, e $150 \mathrm{mg} \mathrm{kg}^{-1}$ de $\mathrm{K}$ como cloreto de potássio. A adubação com micronutrientes foi realizada pela aplicação de solução nutritiva a todos os tratamentos, nas doses: $0,5 \mathrm{mg} \mathrm{kg}^{-1} \mathrm{de} \mathrm{B}$; 1,5 $\mathrm{mg} \mathrm{kg}^{-1}$ de $\mathrm{Cu} ; 3 \mathrm{mg} \mathrm{kg}^{-1}$ de $\mathrm{Zn}$; e $0,1 \mathrm{mg} \mathrm{kg}^{-1}$ de Mo.

As partes aéreas das plantas de milho e soja foram cortadas, respectivamente, aos 42 e 53 dias após a semeadura; foram secas em estufa a $60^{\circ} \mathrm{C}$, pesadas para determinação da massa de matéria seca e moídas em moinho tipo Willey. Após a digestão com ácidos nítrico-perclórico, a atividade do ${ }^{32} \mathrm{P}$ foi determinada em contador de cintilação em meio líquido, por efeito Cerenkov (Vose, 1980), e a concentração de P foi medida conforme Malavolta et al. (1997).

Para verificar se o efeito no aumento da disponibilidade do $\mathrm{P}$ proveniente do $\mathrm{FN}$, aplicado com uma fonte $\mathrm{P}$ de elevada solubilidade em água, foi químico (aumento na solubilização) ou fisiológico (promoção do crescimento inicial das raízes), comparou-se o aproveitamento do $\mathrm{P}$ proveniente do $\mathrm{FN}$, pelas plantas, nos tratamentos em que o FN foi aplicado com o SFT, fixando-se o modo de aplicação (localizado ou não localizado). Assim, para testar se o efeito foi químico, o aproveitamento do P do FN localizado pelas plantas de milho foi comparado com o dos seguintes tratamentos: SFT localizado + FN localizado, SFT não localizado + FN localizado e mistura compactada (localizado). Com o objetivo de testar se o efeito foi fisiológico, o aproveitamento do P do FN não localizado pelo milho foi comparado com o aproveitamento do $\mathrm{P}$ dos tratamentos SFT localizado + FN não localizado e SFT não localizado + FN não localizado. No experimento com a cultura da soja, utilizou-se o tratamento FN não localizado, para comparar com o aproveitamento do $\mathrm{P}$ do $\mathrm{FN}$ das misturas fosfatadas, aplicadas em diferentes modos.

A eficiência agronômica relativa (EAR) foi calculada com os valores de produção de MSPA e P acumulado na MSPA, conforme a seguinte equação: $\operatorname{EAR}(\%)$ $=100\left[\left(\mathrm{Y}_{1}-\mathrm{Y}_{0}\right) /\left(\mathrm{Y}_{2}-\mathrm{Y}_{1}\right)\right]$, em que: $\mathrm{Y}_{0}$ é a produção de MSPA ou $\mathrm{P}$ acumulado na MSPA, no tratamento testemunha (sem aplicação $\mathrm{P}$ ); $\mathrm{Y}_{1}$ é a produção de MSPA ou P acumulado na MSPA, nos tratamentos FN não localizado ou localizado, SFT não localizado e nas misturas de SFT + FN; $\mathrm{Y}_{2}$ é a produção de MSPA ou $\mathrm{P}$ acumulado na MSPA, no tratamento padrão (SFT localizado).

As análises estatísticas da MSPA produzida, de P acumulado na MSPA e dos valores de aproveitamento do P do FN pelas plantas de milho e soja foram realizadas pela análise de variância, e as médias foram comparadas pelo teste de Duncan, a 5\% de probabilidade. O aproveitamento do P total e do SFT por essas culturas foi analisado por contrastes e teste F (SAS Institute, 2001). As análises por contrastes foram realizadas para comparar a eficiência (aproveitamento do $\mathrm{P}$ total) das misturas de fontes fosfatadas, em relação ao tratamento padrão (SFT localizado). 


\section{Resultados e Discussão}

O fornecimento de $\mathrm{P}$ aumentou a produção de MSPA e o P acumulado na MSPA das plantas de milho e de soja (Tabela 1), independentemente da fonte fosfatada e do modo de aplicação utilizados, exceto quando o FN foi aplicado localizadamente ao solo, para as plantas de milho. Neste caso, a produção de MSPA e o P acumulado na MSPA não diferiram do tratamento testemunha.

Os fosfatos sedimentares reativos, como o de Gafsa, apesar de apresentar maior solubilidade do que os fosfatos naturais de origem ígnea ou metamórfica, também apresentam baixa eficiência quando aplicados de modo localizado ao solo (Corrêa et al., 2005).

Resende et al. (2006) aplicaram o fosfato natural reativo de Arad a lanço, em área total e incorporado ou localizado no sulco. Esses autores observaram maior produtividade de grãos de milho quando o fosfato de Arad foi espalhado e incorporado, em comparação ao aplicado localizadamente. Soares et al. (2000) avaliaram a eficiência do fosfato natural de Gafsa farelado em recuperação de pastagem degradada, e observaram resposta superior em produção de matéria seca de Brachiaria decumbens quando esse fosfato foi

Tabela 1. Efeito do modo de aplicação das fontes de fósforo sobre a produção de massa de matéria seca da parte aérea (MSPA), sobre o P acumulado na MSPA (Pac) e sobre a eficiência agronômica relativa (EAR) das fontes, em plantas de milho e de soja ${ }^{(1)}$.

\begin{tabular}{|c|c|c|c|c|}
\hline Tratamento & $\begin{array}{c}\text { MSPA } \\
\text { (g por vaso) }\end{array}$ & $\begin{array}{l}\text { EAR } \\
(\%) \\
\end{array}$ & $\begin{array}{c}\text { Pac } \\
\text { (g por vaso) }\end{array}$ & $\begin{array}{c}\text { EAR } \\
(\%) \\
\end{array}$ \\
\hline & \multicolumn{4}{|c|}{ Plantas de milho } \\
\hline Testemunha & $1,95 \mathrm{~d}$ & - & $3,33 \mathrm{e}$ & - \\
\hline SFT $_{\text {localizado }}$ & $10,24 \mathrm{a}$ & 100 & $19,99 \mathrm{a}$ & 100 \\
\hline $\mathrm{SFT}_{\text {não localizado }}$ & $8,20 \mathrm{~b}$ & 75 & $15,21 \mathrm{bc}$ & 75 \\
\hline $\mathrm{FN}_{\text {localizado }}$ & $3,04 d$ & 13 & $4,83 \mathrm{e}$ & 9 \\
\hline $\mathrm{FN}_{\text {não localizado }}$ & $5,91 \mathrm{c}$ & 48 & $12,18 \mathrm{~d}$ & 53 \\
\hline $\mathrm{SFT}_{\text {localizado }}+\mathrm{FN}_{\text {localizado }}$ & $8,69 b$ & 81 & $14,01 \mathrm{c}$ & 64 \\
\hline $\mathrm{SFT}_{\text {localizado }}+\mathrm{FN}_{\text {não localizado }}$ & $9,08 \mathrm{~b}$ & 86 & $16,24 b$ & 78 \\
\hline $\mathrm{SFT}_{\text {não localizado }}+\mathrm{FN}_{\text {localizado }}$ & $7,84 b$ & 71 & $12,26 \mathrm{~d}$ & 54 \\
\hline $\mathrm{SFT}_{\text {não localizado }}+\mathrm{FN}_{\text {não localizado }}$ & $8,36 b$ & 77 & $13,91 \mathrm{c}$ & 64 \\
\hline Mistura compactada & $8,32 b$ & 77 & $14,38 \mathrm{c}$ & 66 \\
\hline \multirow[t]{2}{*}{ CV $(\%)$} & 10,72 & & 8,92 & \\
\hline & \multicolumn{4}{|c|}{ Plantas de soja } \\
\hline Testemunha & $4,89 \mathrm{~d}$ & - & $5,11 \mathrm{e}$ & - \\
\hline $\mathrm{SFT}_{\text {localizado }}$ & $10,79 a$ & 100 & $22,12 \mathrm{a}$ & 100 \\
\hline $\mathrm{FN}_{\text {não localizado }}$ & $7,30 \mathrm{c}$ & 41 & $11,52 \mathrm{~d}$ & 38 \\
\hline $\mathrm{SFT}_{\text {localizado }}+\mathrm{FN}_{\text {localizado }}$ & $9,24 b$ & 74 & $13,79 \mathrm{c}$ & 51 \\
\hline $\mathrm{SFT}_{\text {localizado }}+\mathrm{FN}_{\text {não localizado }}$ & $10,59 a$ & 97 & $17,90 \mathrm{~b}$ & 75 \\
\hline Mistura compactada & $10,68 \mathrm{a}$ & 98 & $17,29 \mathrm{~b}$ & 72 \\
\hline CV $(\%)$ & 7,85 & & 7,75 & \\
\hline
\end{tabular}

espalhado e incorporado ao solo, em relação ao não incorporado.

A aplicação localizada do SFT resultou em resposta superior à sua aplicação não localizada ao solo, em termos de produção de MSPA e $\mathrm{P}$ acumulado na MSPA, em plantas de milho. O maior contato desse fertilizante de elevada solubilidade com o solo resultou, provavelmente, em maior adsorção e precipitação, conforme relatado por Novais \& Smyth (1999) e Havlin et al. (2005). No entanto, Resende et al. (2006) e Oliveira Junior et al. (2008) não observaram diferenças entre as formas de aplicação do SFT, na produtividade de grãos nas culturas de milho e soja, respectivamente.

Em relação ao $\mathrm{FN}$, o maior contato com o solo proporcionou resposta superior em relação à aplicação localizada dessa fonte de $\mathrm{P}$, quanto à produção de MSPA e acúmulo de P na MSPA de plantas de milho (Tabela 1). Esse resultado indica que o maior contato com o solo proporcionou aumento na solubilização do $\mathrm{FN}$ e na disponibilidade de P para as plantas (Chien \& Menon, 1995a, 1995b; Rajan et al., 1996; Oliveira Junior et al., 2008) e, consequentemente, maior absorção do $\mathrm{P}$ proveniente do $\mathrm{FN}$ pelas plantas, em razão do maior contato das raízes com o FN (Khasawneh \& Doll, 1978).

O efeito do modo de aplicação do SFT ou do FN foi comprovado pelos resultados de aproveitamento do P dessas fontes pelas plantas de milho. A aplicação localizada resultou em maior aproveitamento do $\mathrm{P}$ do SFT do que a não localizada (Tabela 2). A aplicação

Tabela 2. Efeito do modo de aplicação das fontes fosfatadas sobre o aproveitamento do fósforo total e do fósforo proveniente do superfosfato triplo (SFT), em plantas de milho e de soja ${ }^{(1)}$.

\begin{tabular}{|c|c|c|}
\hline Contraste & $\begin{array}{c}\text { Aproveitamento } \\
\text { total }(\%)\end{array}$ & $\begin{array}{c}\text { Aproveitamento } \\
\text { SFT }(\%)\end{array}$ \\
\hline & \multicolumn{2}{|c|}{ Plantas de milho } \\
\hline & $9,75 \times 7,04 * *$ & $9,75 \times 7,04 * *$ \\
\hline $\mathrm{FN}_{\text {localizado }} \mathrm{x} \mathrm{FN}_{\text {não localizado }}$ & $0,49 \times 3,80 * *$ & $-(2)$ \\
\hline $\mathrm{SFT}_{\text {localizado }} \mathrm{x}$ todas misturas & $9,75 \times 5,80 * *$ & $9,75 \times 8,70^{\mathrm{ns}}$ \\
\hline $\mathrm{SFT}_{\text {localizado }} \times \mathrm{SFT}_{\text {localizado }}+\mathrm{FN}_{\text {localizado }}$ & $9,75 \times 5,95 * *$ & $9,75 \times 10,54^{\text {ns }}$ \\
\hline $\mathrm{SFT}_{\text {localizado }} \times \mathrm{SFT}_{\text {localizado }}+\mathrm{FN}_{\text {não localizado }}$ & $9,75 \times 7,22 * *$ & $9,75 \times 10,25^{\text {ns }}$ \\
\hline $\mathrm{SFT}_{\text {localizado }} \times \mathrm{SFT}_{\text {não localizado }}+\mathrm{FN}_{\text {localizado }}$ & $9,75 \times 4,59 * *$ & $9,75 \times 8,04^{*}$ \\
\hline $\mathrm{SFT}_{\text {localizado }} \times \mathrm{SFT}_{\text {não localizado }}+\mathrm{FN}_{\text {não localizado }}$ & $9,75 \times 5,01 * *$ & $9,75 \times 6,78 * *$ \\
\hline \multirow[t]{2}{*}{ SFT $_{\text {localizado }} \mathrm{x}$ mistura compactada localizado } & $9,75 \times 6,20 * *$ & $9,75 \times 7,89^{*}$ \\
\hline & \multicolumn{2}{|c|}{ Plantas de soja } \\
\hline $\mathrm{SFT}_{\text {localizado }} \mathrm{x}$ todas misturas & $9,62 \times 7,03 * *$ & $9,62 \times 10,18^{\text {ns }}$ \\
\hline $\mathrm{SFT}_{\text {localizado }} \times \mathrm{SFT}_{\text {localizado }}+\mathrm{FN}_{\text {localizado }}$ & $9,62 \times 5,91 * *$ & $9,62 \times 10,16^{\mathrm{ns}}$ \\
\hline $\mathrm{SFT}_{\text {localizado }} \times \mathrm{SFT}_{\text {localizado }}+\mathrm{FN}_{\text {não localizado }}$ & $9,62 \times 7,71 * *$ & $9,62 \times 10,35^{\text {ns }}$ \\
\hline SFT $_{\text {localizado }} \times$ mistura compactada localizado & $9,62 \times 7,47 * *$ & $9,62 \times 10,05^{\mathrm{ns}}$ \\
\hline
\end{tabular}


não localizada do FN proporcionou aproveitamento do $P$ pelo milho superior em sete vezes ao aproveitamento resultante da aplicação localizada (Tabela 3 ).

A aplicação localizada do SFT proporcionou maior produção de MSPA, acúmulo de P na MSPA, e maior aproveitamento do $\mathrm{P}$ dessa fonte pelas plantas de milho e soja (Tabelas 1 e 2). Assim, em ambos os experimentos, este tratamento foi utilizado como padrão nas comparações relativas ao aproveitamento do P total e do proveniente do SFT e das misturas de SFT e FN pelas plantas de milho e soja.

Não houve diferença na produção de MSPA das plantas de milho entre os tratamentos com as misturas das duas fontes fosfatadas e o tratamento SFT não localizado (Tabela 1). Para a MSPA, foram observados valores de EAR na faixa de 70 a $80 \%$ nesses tratamentos. Considerando-se o $\mathrm{P}$ acumulado na MSPA, as misturas de SFT e FN proporcionaram valores de EAR entre 50 a $80 \%$.

A retenção de $\mathrm{P}$ do SFT em solos altamente intemperizados, favorecida pelo maior contato com o solo no tratamento não localizado, e a menor solubilização do $\mathrm{P}$ do $\mathrm{FN}$ aplicado localizadamente ao solo resultaram na baixa EAR (em termos de P acumulado) observada nos tratamentos com a mistura SFT não localizado + FN localizado (54\%), que foi equivalente à do tratamento FN não localizado (53\%).

A aplicação do SFT localizado resultou em resposta na produção de MSPA das plantas de soja superior à da mistura SFT localizado + FN localizado, mas não diferiu da mistura compactada e do tratamento SFT localizado + FN não localizado (Tabela 1). Os valores de $\mathrm{P}$ acumulado na MSPA das plantas de soja, nos tratamentos com as misturas entre SFT e FN, foram

Tabela 3. Aproveitamento, pelas plantas de milho ou de soja, do fósforo proveniente do fosfato natural reativo de Gafsa (FN) aplicado isoladamente ou em misturas com superfosfato triplo (SFT), em diferentes métodos de aplicação ${ }^{(1)}$.

\begin{tabular}{lcc}
\hline Tratamento & \multicolumn{2}{c}{ Aproveitamento (\%) } \\
\cline { 2 - 3 } & Milho & Soja \\
\hline $\mathrm{FN}_{\text {localizado }}$ & $0,49 \mathrm{~b}$ & - \\
$\mathrm{SFT}_{\text {não localizado }}+\mathrm{FN}_{\text {localizado }}$ & $1,15 \mathrm{~b}$ & - \\
$\mathrm{SFT}_{\text {não localizado }}+\mathrm{FN}_{\text {não localizado }}$ & $3,24 \mathrm{a}$ & - \\
$\mathrm{SFT}_{\text {localizado }}+\mathrm{FN}_{\text {localizado }}$ & $1,37 \mathrm{~b}$ & $1,65 \mathrm{c}$ \\
$\mathrm{FN}_{\text {não localizado }}$ & $3,80 \mathrm{a}$ & $3,32 \mathrm{~b}$ \\
$\mathrm{Mistura}$ compactada & $4,02 \mathrm{a}$ & $4,88 \mathrm{a}$ \\
$\mathrm{SFT}_{\text {localizado }}+\mathrm{FN}_{\text {não localizado }}$ & $4,18 \mathrm{a}$ & $5,06 \mathrm{a}$ \\
\hline
\end{tabular}

(1)Médias seguidas de letras iguais, nas colunas, não diferem pelo teste de Duncan, a 5\% de probabilidade. - Não avaliado. inferiores ao do tratamento SFT localizado. Os valores de EAR, calculados com base na produção de MSPA das plantas de soja, dos tratamentos SFT localizado + FN não localizado (97\%) e mistura compactada (98\%) foram próximos aos valores do tratamento SFT localizado. Motomiya et al. (2004) observaram menor EAR das misturas entre as fontes de superfosfato triplo e fosfato de Gafsa, nas proporções $67 \% \mathrm{SFT}+33 \% \mathrm{FN}$ e $33 \%$ SFT $+67 \%$ FN, em relação ao SFT localizado.

$\mathrm{O}$ aproveitamento do $\mathrm{P}$ do SFT localizado foi superior ao de todas as misturas entre fontes de $\mathrm{P}$, tanto nas plantas de milho e como nas de soja (Tabela 2). Horowitz \& Meurer (2003) também observaram maior eficiência do SFT em fornecer P para as plantas em dois cultivos de milho, com duração de 21 dias cada, em comparação aos fosfatos naturais reativos de Gafsa e de Gantour Black. No entanto, Soares et al. (2000) observaram a mesma eficiência entre o SFT e fosfato reativo de Gafsa espalhado e incorporado, na produção de matéria seca de Brachiaria decumbens, em experimento de campo com aproximadamente um ano de duração.

$\mathrm{Na}$ comparação do tratamento $\mathrm{FN}$ localizado com os tratamentos com a mistura compactada e SFT localizado + FN localizado, observou-se que somente na mistura compactada houve aumento no aproveitamento do $\mathrm{P}$ proveniente do $\mathrm{FN}$, pelas plantas de milho (Tabela 3). Isso demonstra que a interação química entre essas fontes de P no grânulo do fertilizante deve ter promovido a solubilização do $\mathrm{P}$ presente no $\mathrm{FN}$ e, consequentemente, o aumento na disponibilidade do $\mathrm{P}$ proveniente do $\mathrm{FN}$. O aumento na solubilização ocorre, principalmente, em razão da acidez gerada pela hidrólise do fosfato no fertilizante SFT (Mokwunye \& Chien, 1980). No entanto, a promoção no aproveitamento do $\mathrm{P}$ proveniente do $\mathrm{FN}$ não foi observada quando as fontes foram aplicadas em contato físico no solo, mas não no mesmo grânulo.

Não se observou diferença no aproveitamento, pelas plantas de milho, do $\mathrm{P}$ do $\mathrm{FN}$ no tratamento SFT localizado + FN não localizado, em relação ao tratamento FN não localizado (Tabela 3 ).

No entanto, no segundo experimento, quando as plantas de soja foram utilizadas como cultura teste, observou-se que o aproveitamento do $\mathrm{P}$ do $\mathrm{FN}$ foi superior no tratamento SFT localizado + FN não localizado, em comparação ao tratamento $\mathrm{FN}$ não localizado (Tabela 3). Isso indica que aplicação 
localizada do STF provavelmente promoveu o desenvolvimento do sistema radicular (parâmetro não avaliado nos experimentos) das plantas de soja (efeito fisiológico), o que teria possibilitado maior absorção do $\mathrm{P}$ proveniente do FN, conforme verificado por Chien et al. (1996) e Zapata \& Zaharah (2002).

Os fosfatos naturais são mais eficientes em leguminosas do que em cereais, pois a eficiência relativa do fosfato natural é superior em plantas com menor demanda de concentração de $\mathrm{P}$ na solução do solo, que é o caso das leguminosas em relação aos cereais. (Chien \& Menon, 1995b). Esse fato contribui para explicar a diferença entre os resultados observados nos experimentos com milho e soja neste trabalho.

\section{Conclusões}

1. A aplicação localizada do superfosfato triplo aumenta a eficiência dessa fonte no fornecimento de fósforo às plantas.

2. A aplicação do fosfato natural reativo de modo não localizado no solo aumenta a eficiência dessa fonte no fornecimento de fósforo às plantas.

3. O aproveitamento do fósforo do fosfato natural reativo de Gafsa, pelas plantas de milho e soja, aumenta quando o fósforo é aplicado com o superfosfato triplo, e esse efeito depende do método de aplicação dessas fontes fosfatadas no solo.

\section{Referências}

CAMARGO, O.A. de; MONIZ, A.C.; JORGE, J.A.; VALADARES, J.M.A.S. Métodos de análise química, mineralógica e física de solos. Campinas: Instituto Agronômico, 1986. 94p. (IAC. Boletim Técnico, 106).

CHIEN, S.H.; MENON, R.G. Agronomic evaluation of modified phosphate rock products. IFDC's experience. Fertilizer Research, v.41, p.197-209, 1995a.

CHIEN, S.H.; MENON, R.G. Factors affecting the agronomic effectiveness of phosphate rock for direct application. Fertilizer Research, v.41, p.227-234, 1995b.

CHIEN, S.H.; MENON, R.G.; BILLINGHAM, K.S. Phosphorus availability from phosphate rock as enhanced by water-soluble phosphorus. Soil Science Society of America Journal, v.60, p.1173-1177, 1996.

COMPANHIA NACIONAL DE ABASTECIMENTO. Acompanhamento da safra brasileira: grãos safra 2007/2008: décimo segundo levantamento: setembro de 2008. Brasília: Conab, 2008. Disponível em: <http://www.conab.gov.br/conabweb/ download/safra/12_levantamento_set2008.pdf $>$. Acesso em: 20 dez. 2008.
CORRÊA, R.M.; NASCIMENTO, C.W.A. do; SOUZA, S.K. de S; FREIRE, F.J.; SILVA, G.B. da. Gafsa rock phosphate and triple superphosphate for dry matter production and $\mathrm{P}$ uptake by corn. Scientia Agricola, v.62, p.159-164, 2005.

FRANZINI, V.I.; MURAOKA, T.; MENDES, F.L. Ratio and rate effects of ${ }^{32} \mathrm{P}$-triple superphosphate and phosphate rock mixtures on corn growth. Scientia Agricola, v.66, p.71-76, 2009.

GOEDERT, W.J.; SOUSA, D.M.G. Uso eficiente de fertilizantes fosfatados. In: SIMPÓSIO SOBRE FERTILIZANTES NA AGRICULTURA BRASILEIRA, 1., 1984, Brasília. Anais. Brasília: Embrapa-DEP, 1984. p.255-289. (Embrapa-DEP. Documentos, 14).

HAVLIN, J.L.; BEATON, J.D.; TISDALE, S.L.; NELSON, W.L. Soil fertility and fertilizers: an introduction to nutrient management. 7.ed. New Jersey: Pearson, 2005. 515p.

HOROWITZ, N.; MEURER, E.J. Eficiência de dois fosfatos naturais farelados em função do tamanho da partícula. Ciência Rural, v.33, p.41-47, 2003.

KHASAWNEH, F.E.; DOLL, E.C. The use of phosphate rock for direct application to soils. Advances in Agronomy, v.30, p.159-206, 1978.

MALAVOLTA, E.; VITTI, G.C.; OLIVEIRA, S.A. Avaliação do estado nutricional das plantas: princípios e aplicações. 2.ed. Piracicaba: Potafos, 1997. 319p.

MOKWUNYE, A.U.; CHIEN, S.H. Reactions of partially acidulated phosphate rock with soils from the tropics. Soil Science Society of America Journal, v.44, p.477-482, 1980.

MOTOMIYA, W.R.; FABRÍCIO, A.C.; MARCHETTI, M.E.; GONÇALVES, M.C.; ROBAINA, A.D.; NOVELINO, J.O. Métodos de aplicação de fosfato na soja em plantio direto. Pesquisa Agropecuária Brasileira, v.39, p.307-312, 2004.

NOVAIS, R.F. de; SMYTH, T.J. Fósforo em solo e planta em condições tropicais. Viçosa: UFV, 1999. 399p.

OLIVEIRA JUNIOR, A. de; PROCHNOW, L.I.; KLEPKER, D. Eficiência agronômica de fosfato natural reativo na cultura da soja. Pesquisa Agropecuária Brasileira, v.43, p.623-631, 2008.

RAIJ, B. van; ANDRADE，J.C. de; CANTARELLA, H.; QUAGGIO, J.A. Análise química para avaliação da fertilidade de solos tropicais. Campinas: Instituto Agronômico, 2001. 285p.

RAIJ, B. van; CANTARELLA, H.; QUAGGIO, J.A; FURLANI, A.M.C. (Ed.). Recomendações de adubação e calagem para o Estado de São Paulo. 2.ed. Campinas: Instituto Agronômico, 1996. 285p. (IAC. Boletim Técnico, 100).

RAJAN, S.S.S.; WATKINSON, J.H.; SINCLAIR, A.G. Phosphate rocks for direct application to soils. Advances in Agronomy, v.57, p.77-159, 1996.

RESENDE, A.V. de; FURTINI NETO, A.E.; ALVES, V.M.C; MUNIZ, J.A.; CURI, N.; FAQUIN, V.; KIMPARA, D.I.; SANTOS, J.Z.L.; CARNEIRO, L.F. Fontes e modos de aplicação de fósforo para o milho em solo cultivado da região do Cerrado. Revista Brasileira de Ciência do Solo, v.30, p.453-466, 2006. 
SANTOS, H.G. dos; JACOMINE, P.K.T.; ANJOS, L.H.C. dos; OLIVEIRA, V.A. de; OLIVEIRA, J.B. de; COELHO, M.R.; LUMBRERAS, J.F.; CUNHA, T.J.F. (Ed.). Sistema brasileiro de classificação de solos. 2.ed. Rio de Janeiro: Embrapa Solos, 2006. $306 \mathrm{p}$.

SAS INSTITUTE. SAS user's guide: statistics. Versão 8.2. Cary: SAS Institute, 2001.

SOARES, W.V.; LOBATO, E.; SOUSA, D.M.G. de; REIN, T.A. Avaliação do fosfato natural de Gafsa para recuperação de pastagem degradada em Latossolo Vermelho-Escuro. Pesquisa Agropecuária Brasileira, v.35, p.819-825, 2000.

SOUSA, D.M.G. de; REIN, T.A.; LOBATO, E. Solubilidade e eficiência agronômica de fosfatos naturais reativos avaliados com a cultura da soja em um Latossolo de Cerrado. In: SIMPÓSIO
NACIONAL DO CERRADO, 9.; SIMPÓSIO INTERNACIONAL SAVANAS TROPICAIS, 2., 2008, Brasília. Desafios e estratégias para o equilíbrio entre sociedade, agronegócio e recursos naturais: anais. Planaltina: Embrapa Cerrados, 2008.

SYERS, J.K.; MACKAY, A.D.; BROWN, M.W.; CURRIE, L.D. Chemical and physical characteristics of phosphate rock materials of varying reactivity. Journal of the Science of Food and Agriculture, v.37, p.1057-1064, 1986.

VOSE, P.B. Introduction to nuclear techniques in agronomy plant biology. London: Pergamon Press, 1980. 391p.

ZAPATA, F.; ZAHARAH, A.R. Phosphorus availability from phosphate rock and sewage sludge as influenced by the addition of water soluble phosphate fertilizer. Nutrient Cycling in Agroecosystems, v.63, p.43-48, 2002.

Recebido em 14 de janeiro de 2009 e aprovado em 27 de junho de 2009 